\title{
ORIGINAL RESEARCH \\ Asymmetric Development of the Hippocampal Region Is Common: A Fetal MR Imaging Study
}

\begin{abstract}
D. Bajic
N. Canto Moreira

J. Wikström

R. Raininko

BACKGROUND AND PURPOSE: Hippocampal development is poorly understood. This study evaluated the normal development of the hippocampal region during the fetal period by using MR imaging.

MATERIALS AND METHODS: MR images of 63 fetuses without intracranial pathology were reviewed independently by 2 radiologists with no knowledge of the fetal GA. Three MR images were performed postmortem and 60 in vivo. The progress of hippocampal inversion was analyzed in coronal sections, and the left and right sides of the hippocampal region were compared in every case.

RESULTS: The fetuses in the postmortem examinations were at GWs 17-18 and in the in vivo examinations, at GWs 19-36. The hippocampal sulcus was open, bi- or unilaterally, in 39 fetuses. The oldest was at GW 32. The sulcus was closed at GW 21 at the earliest, unilaterally. In 26/63 fetuses $(41 \%)$, the deepening or closure of the hippocampal sulcus or hippocampal inversion was asymmetric; in 23 fetuses, the right side developed faster. A shallow collateral sulcus was found earliest at GW 17. A deep collateral sulcus was visible earliest at GW 26 unilaterally, but in all fetuses from GW 31 onward, it was seen bilaterally. The orientation of the collateral sulcus was not related to the GA.

CoNCLUSIONS: There are wide individual temporal variations in the development and the inversion process of the hippocampal sulcus as well as in the formation of the collateral sulcus. Asymmetric development is common, and in most of the asymmetric cases, the right hippocampus develops faster.
\end{abstract}

ABBREVIATIONS: $\mathrm{GA}=$ gestational age, $\mathrm{GW}=$ gestation week, $\mathrm{IHI}=$ incomplete hippocampal inversion

$I_{\text {the }}^{\text {tim }}$ is important to be familiar with normal development, so that normal developmental changes are not confused with abnormalities, particularly in fetuses being assessed for possible termination if an abnormality is found. Knowledge of the hippocampal development may also help us to understand some morphologic deviations found even in adult populations, such as IHI, also called hippocampal malrotation.

There are a growing number of morphologic studies of the fetal hippocampus, but most are microscopic examinations of limited numbers of formalin-fixed aborted fetuses, focusing more on the cellular types and organization than on the general shape of the hippocampus and adjacent structures. Three microscopic studies also included MR images of formalinfixed specimens, ${ }^{1-3}$ but a major weak point of this type of approach is the fact that anatomic shape and proportions usually change when the brain is removed from the skull and placed in the vessels for formalin fixation or on the table for cutting or imaging. Therefore, examination of the brain inside the skull should give more reliable information on the shape of the brain structures.

Hippocampal development begins at GW 8, and the hippocampal sulcus becomes visible at GW $10^{4}$ as a shallow groove on the medial side of the temporal lobe. During fetal development, the hippocampal sulcus, situated between the

\section{Received May 6, 2011; accepted after revision June 24.}

From the Department of Radiology (D.B., N.C.M., J.W., R.R.), Uppsala University, Uppsala, Sweden; and Neuroradiology Section C (N.C.M.), Clinica Dr Campos Costa, Porto, Portugal.

Please address correspondence to Dragan Bajic, MD, PhD, Department of Radiology, University Hospital, 75185 Uppsala, Sweden; e-mail: dragan.bajic@akademiska.se

Indicates open access to non-subscribers at www.ajnr.org

http://dx.doi.org/10.3174/ajnr.A2814 dentate nucleus and cornu ammonis, infolds in the temporal lobe and becomes both deeper and narrower. Thereafter, the hippocampal rotation begins as a part of the inversion process, which has been reported to be completed at approximately GW $21,{ }^{1,2,4-6}$ but according to a recent sonographic study of premature brains, this process seems to take longer. ${ }^{7}$ The fusion of the walls of the hippocampal sulcus continues until GW 30, ${ }^{4}$ but focal open remnants may be found even in adult brains. ${ }^{8}$ The orientation of the hippocampal sulcus may also change during the inversion process.

Cerebral sulcal development has been described in vivo in a large fetal MR series ranging from GW 22 to GW $38 .^{9}$ In that study, the hippocampal sulcus could be seen at GWs 22-23 and the collateral sulcus, at GWs 23-26. There is only 1 in vivo fetal MR imaging study focusing on the hippocampi, ${ }^{10}$ and it demonstrated a progressive change of the hippocampal infolding angle (the angle between the line connecting the lateral margin of the cornu ammonis with the medial superior margin of the subiculum and the line passing midline structures) from GW 20 to GW 37.

The general shape of the hippocampus has not been assessed in the above-mentioned studies. The fully inverted hippocampus is oval with a horizontal long axis in coronal sections. Baker and Barkovich ${ }^{11}$ have presented a hypothesis that this oval shape is preceded by a round or pyramidal shape. The nonoval shape with an indefinite or vertical long axis can be seen in figures showing microscopic specimens of fetuses. ${ }^{4-6}$ The long axis in coronal sections of the hippocampus was never mentioned in the previous studies. The nonoval shape was revealed more often in younger than older premature neonates in a sonographic study. ${ }^{7}$ However, the nonoval shape can be seen throughout life in $19 \%$ of the general population, most often on the left side. ${ }^{12,13}$ 
The aim of this study was to evaluate the development of the hippocampal region in fetuses by using MR imaging.

\section{Materials and Methods}

The brain images from postmortem MR images of 12 nonfixed aborted fetuses that were examined before autopsy and from 306 in vivo clinical fetal MR images were assessed. The postmortem examinations were performed in Sweden, and the in vivo examinations, in Sweden or Portugal. All the fetuses that underwent postmortem examinations and in which CNS pathology was revealed at autopsy or MR imaging were excluded. The indications for clinical fetal MR imaging were the following: suspected body malformations, CNS abnormalities suspected on previous sonography, or abnormal findings on screening laboratory tests. All subjects were excluded from the study if repeated sonography or clinical studies revealed brain pathology as was the case with fetuses showing deviating MR imaging findings. The most common of such deviating findings was widening of the lateral ventricles, and fetuses with atrial diameter wider than $10 \mathrm{~mm}$ were excluded. ${ }^{14-17}$ All examinations in which the hippocampal regions were not well assessed bilaterally or in which the coronal sections were not symmetrically positioned were also excluded (180 fetuses, all examined in vivo). In the end, 3 postmortem examinations and 60 in vivo examinations were accepted for the final analysis. Two of the 3 postmortem examinations were performed within 5 hours after termination. The third fetus was kept in a cooler while waiting for an MR imaging examination 2 days later. The brains were not removed from the skulls.

The GA at the moment of the MR imaging examination was based on sonographic data ${ }^{18}$ and defined as the number of complete GWs.

The hippocampal regions were assessed in $\geq 1$ coronal section through the area. In the younger fetuses, the hippocampus could only be evaluated if it was situated in 1 section and not shared in 2 . The progress of the hippocampal inversion was analyzed, and a comparison between the right and left side was made in every case. The side of the heart was regarded as the left side of the fetus. As additional visual references, we used the liver and the gastric chamber. Combined, it was always possible to distinguish the right from the left side on coronal sections. In cases in which the hippocampal sulcus was not yet closed (Figs 1 and $2 A$ ), the angle between the upper and lower lip of the hippocampal sulcus was assessed. If the hippocampal sulcus was already closed, the shape of the inverted hippocampus was classified as nonoval (round, pyramidal, or near-oval with a vertical long axis) (Fig $2 B$ ) or oval with a horizontal long axis (Fig $2 C$ ). The nonoval hippocampal shape was classified as less developed than the oval shape according to the hypothesis proposed by Baker and Barkovich. ${ }^{11}$ The presence and orientation of the collateral sulcus was also recorded. If the angle between the axis of the collateral sulcus and the horizontal axis of the hippocampus was $>70^{\circ}$, the collateral sulcus was defined as vertical. ${ }^{12}$

One experienced radiologist evaluated all 318 examinations and proposed exclusions according to the criteria presented above. The examinations accepted for the final analysis (63 examinations, 126 hippocampal regions) were then also evaluated by another experienced radiologist independently. Both radiologists were blinded to GAs. Interobserver agreement in the assessment of the hippocampal structures was $90 \%$, and in assessment of the collateral sulcus, $97 \%$. In cases of disagreement, the radiologists reassessed the images and a consensus was achieved.

The studies were performed with Intera scanners (Philips Healthcare, Best, the Netherlands) operating at 1.5T. The postmortem ex-

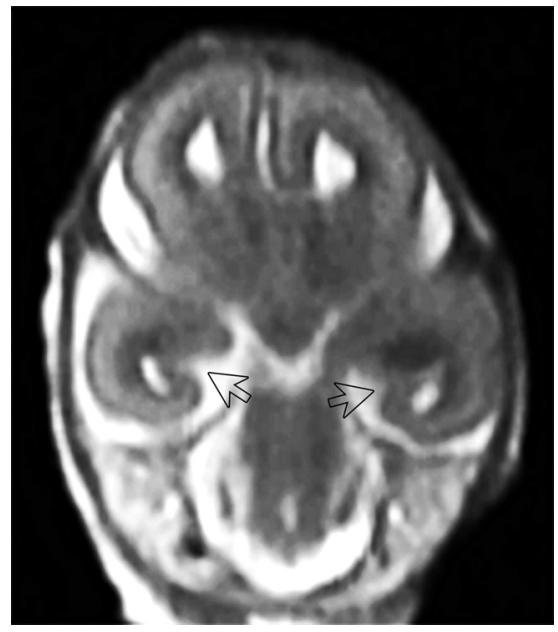

Fig 1. Postmortem MRI study of a fetus aborted at $17 \mathrm{GW}$. The arrows indicate widely open symmetric hippocampal sulci. T2-weighted TSE coronal image.

aminations were performed by using a birdcage knee-foot coil. T2weighted 2D FSE sequences were obtained in the sagittal, coronal, and transverse planes, with a section thickness of $2 \mathrm{~mm}$ and in-plane resolution of $0.50 \times 0.53 \mathrm{~mm}$ (transverse plane) and $0.59 \times 0.62 \mathrm{~mm}$ (coronal and sagittal planes). A T1-weighted magnetization-prepared 3D gradient recalled-echo sequence was performed in the sagittal plane, with a section thickness of $1 \mathrm{~mm}$ and an in-plane resolution of $0.59 \times 0.91 \mathrm{~mm}$. From the acquired T1-weighted images, reformats were constructed in the axial and coronal planes. The in vivo studies were performed by using abdominal phased-array flexible coils. The imaging protocol included T2-weighted single-shot FSE sequences in the sagittal, axial, and coronal planes. The section thickness was 3-4 $\mathrm{mm}$, and the matrix, between $256 \times 256$ and $364 \times 256$. FOVs ranged from $20 \times 20$ to $30 \times 30 \mathrm{~cm}$. No sedation was given to the mothers.

The study protocol was approved by the local ethics committees in Sweden and in Portugal. In Sweden, all mothers gave written informed consent before the examinations. In Portugal, the ethics committee allowed retrospective analysis of clinical studies in the project without the mothers' consent.

The statistical packages JMP (Version 5.1, http://www.jmp.com) and StatsDirect 2.5.7 (http://www.statsdirect.com) were used. Differences in proportions were analyzed by the likelihood ratio $\chi^{2}$ method. $P$ values $<.05$ were considered significant. All tests were 2-tailed.

\section{Results}

The 3 postmortem examinations were performed at GWs 1718 , and the 60 in vivo examinations, at GWs 19-36.

All fetuses had an identifiable hippocampal sulcus bilaterally. The hippocampal sulcus was open, bilaterally or unilaterally, in 39 of the 51 fetuses that were examined from GW 17 to GW 32 (Fig 3). Of these, 35 had a bilaterally open hippocampal sulcus, but in $21 / 35$, the angle between the upper and lower lip was already $<90^{\circ}$ on both sides. In 5 fetuses, the angle was $<90^{\circ}$ on the right side but $>90^{\circ}$ on the left side, and in $9,>90^{\circ}$ bilaterally.

A closed hippocampal sulcus was found in 28 fetuses (Fig 3). In 4 fetuses, from GW 21 to GW 30, the right sulcus was closed but the left one was still open. The hippocampal sulcus was bilaterally closed from GW 33 onward in all cases. Asymmetry was observed in the closing process in 11 fetuses in which both hippocampal sulci still were open (Fig 4). The 

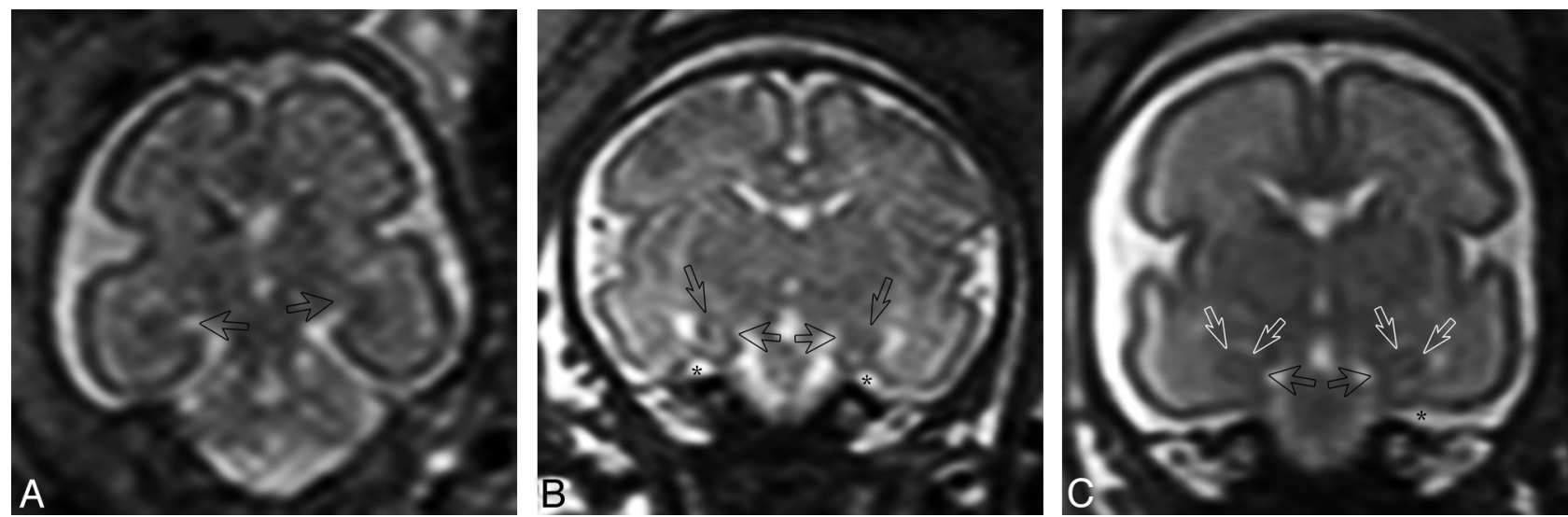

Fig 2. In vivo T2-weighted single-shot FSE coronal images. A, Fetus at GW 25. The arrows indicate the hippocampal sulci. The hippocampal sulcus is larger on the left side. $B$, Fetus at GW 29. Closed hippocampal sulcus and a nonoval hippocampus with a vertical long axis bilaterally. The arrows indicate the hippocampal region, and the stars, the collateral sulcus. $C$, Fetus at GW 29. Closed hippocampal sulcus and an oval hippocampus with a horizontal long axis bilaterally. White arrows indicate the hippocampal region; the black arrows, the closed hippocampal sulcus; and the star, the collateral sulcus.

\section{Number of fetuses}

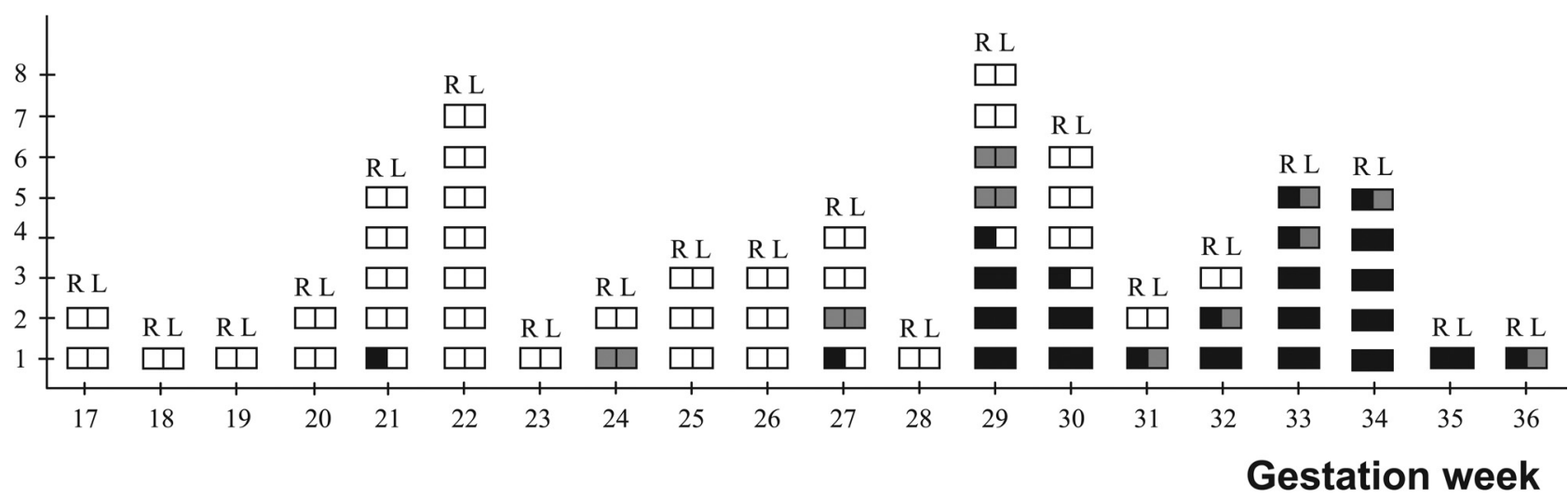

Fig 3. Closure of the hippocampal sulcus and the shapes of the hippocampi in fetuses with a closed hippocampal sulcus. Each square pair describes a fetus. $R$ indicates right side; $L$, left side; open square, open hippocampal sulcus; shaded square, nonoval shape of the inverted hippocampus; black square, oval shape of the inverted hippocampus, with the long axis horizontal (complete inversion).

narrower hippocampal sulcus was found on the right side in 8 fetuses and on the left side in 3 fetuses.

Concerning hippocampal shape, the distribution of the symmetric and asymmetric findings is shown in Fig 3. The youngest fetus with an open hippocampal sulcus on 1 side and a closed one on the other side had an oval shape in the already inverted hippocampus at GW 21, but in 3 older fetuses at GWs 27-30, the shape was nonoval on the closed side. Nonoval hippocampal shapes could be found bilaterally at GWs 24-29 and unilaterally with the oval shape on the contralateral side at GWs 31-36. In the 12 fetuses of GW 33 or more, when all the hippocampal sulci were closed, the shape of the hippocampus was still asymmetric in 4 (33\%), being nonoval on the left side and oval on the right.

A summary of the symmetric and asymmetric findings is seen in Fig 4. Asymmetric hippocampal development was found in 26/63 fetuses (41\%). The development was faster on the right in 23 fetuses and on the left only in 3.

The development of the collateral sulcus is shown in Fig 5. A shallow collateral sulcus was detected at the earliest in 1 fetus at GW 17, but in 1 subject, this sulcus could not be seen even at week 29. A deep well-defined collateral sulcus was seen at the earliest at GW 26 on the right and at GW 27 on the left. All 17 fetuses imaged at GW 31 or later had developed a deep collateral sulcus bilaterally. In fourteen of 24 fetuses that presented a bilateral deep collateral sulcus, there was a uni- or bilateral vertical orientation (58\%). The collateral sulcus was vertical in $5 / 26$ fetuses (19\%) on the right side and in 13/25 fetuses (52\%) on the left side, a difference that was statistically significant $(P=.014)$. In 9 fetuses, a nonoval hippocampal shape was associated with a deep collateral sulcus. In 6 of these cases, the adjacent collateral sulcus was vertical.

\section{Discussion}

This is the second prenatal MR imaging study focused on the hippocampus ${ }^{10}$ and the first in which gyral development in the hippocampal region and the shape of the inverted hippocampus have been assessed. We found that there are wide individual temporal variations in the deepening and closure of the hippocampal sulcus and in the development of the collateral sulcus, which also showed different types of orientation. After the closure of the hippocampal sulcus, the inverted hippocampus could be a nonoval shape (Fig $2 B$ ) or alternatively an oval shape with a near-horizontal long axis (Fig 2C). This last- 


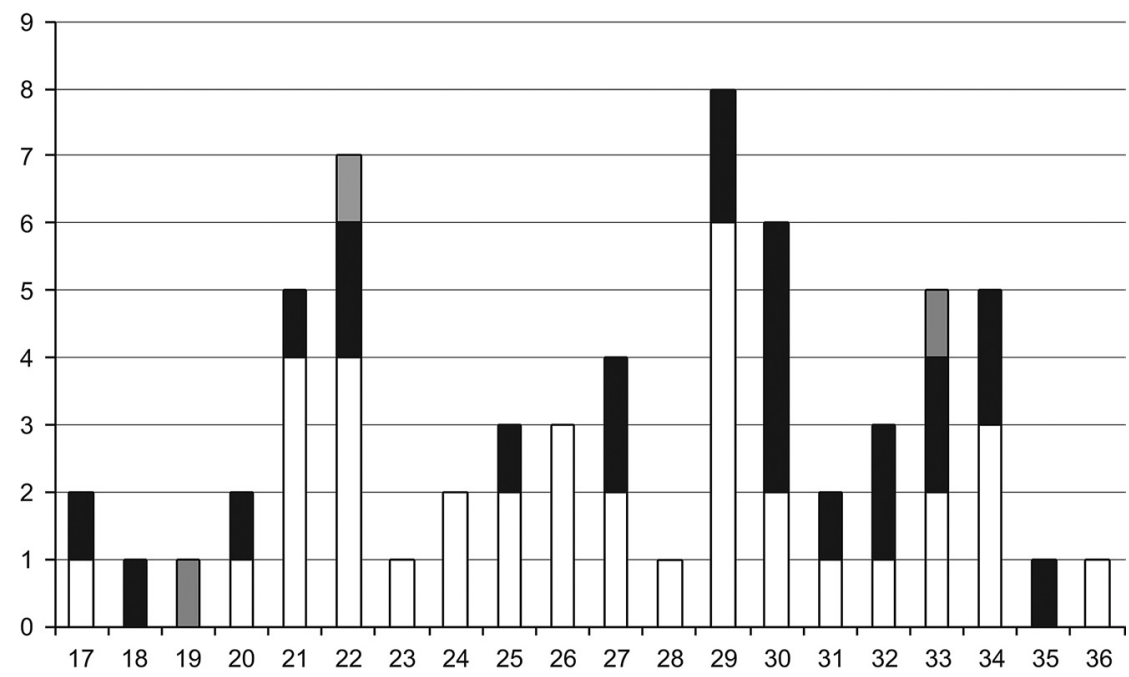

Gestation week

Fig 4. Comparison of the development of the left and right sides of the hippocampus. Open box indicates symmetric development; shaded square, the left side more developed; black square, the right side more developed.

\section{Number of fetuses}

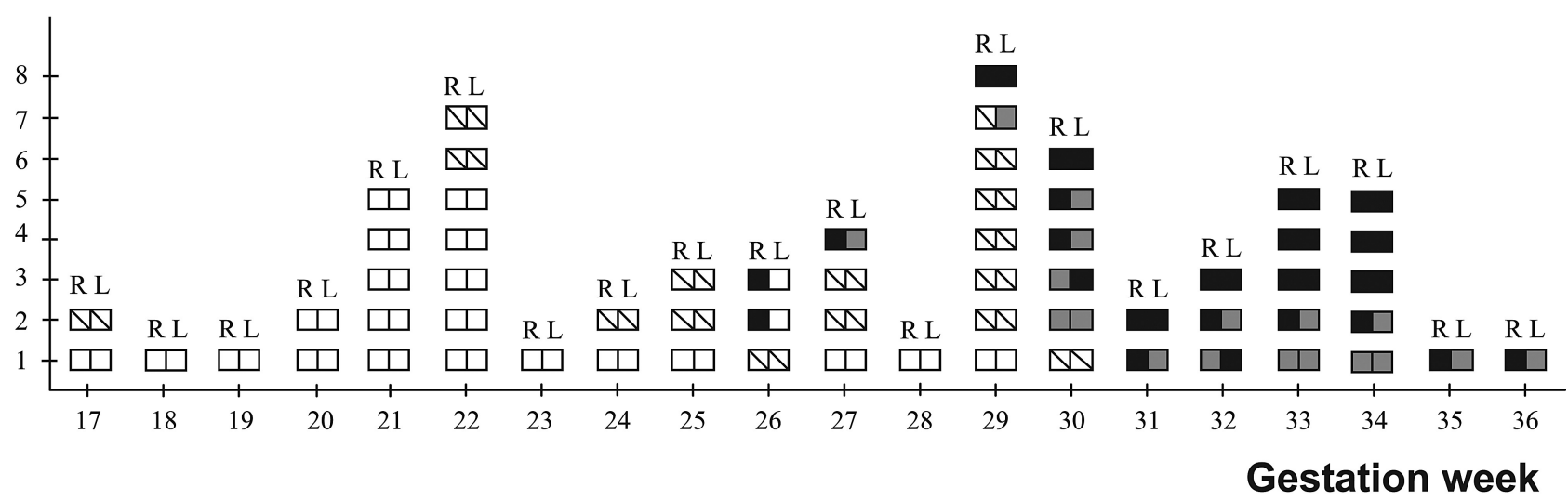

Fig 5. Collateral sulcus at different GAs. Each square pair describes a fetus. R indicates right side; L, left side. Collateral sulcus: Open square indicates not visible; slashed square, shallow; shaded square, vertical; black square, oblique or horizontal.

shape represents complete hippocampal inversion. The development of the hippocampus, including the hippocampal sulcus and the inverted hippocampal formation, was often asymmetric $(26 / 63=41 \%)$, and in a great majority of the asymmetric cases, the right side $(23 / 26=88 \%)$ had developed faster. Also the collateral sulcus developed earlier on the right side. The orientation of the collateral sulcus can be vertical or horizontal; horizontal is the more common type in adults. The vertical orientation was significantly more common on the left side.

It is not easy to discern small structures such as the hippocampus on fetal MR imaging. Image quality and resolution were better in the postmortem images (Fig 1), in which there are no movement artifacts or limitations of examination time. The hippocampal region was well assessed in all 12 postmortem examinations at GWs $17-19$, but in vivo, the image quality was much lower (Fig $2 A-C$ ), and $59 \%$ of the examinations available in the data base were rejected for technical reasons. The youngest living fetus with a resolution good enough for hippocampal evaluation was examined at GW 19.

The hippocampal sulcus was identifiable in all of the fetuses in our series, in good accordance with the formalin-fixed brain series at the same GAs, ${ }^{1-3}$ but earlier than that described in a previously published fetal MR imaging study, ${ }^{9}$ a feature that might be related to the more recent imaging techniques that we used.

Concerning hippocampal shape, studies on the formalinfixed brain indicate that the hippocampus should have reached an adult appearance by GW $21,{ }^{1,2,4-6}$ but in our study, we could detect a closed hippocampal sulcus in only 1 fetus at that age, and open hippocampal sulci could still be seen at up to GW 32, thus reflecting a larger time variation for closure than previously thought.

When a fetus has a closed hippocampal sulcus, the hip- 
pocampus is more or less inverted, and we classified its shape by using the same criteria as in earlier studies on adults, children, and premature neonates. ${ }^{7,12,13}$ Most of the population has an oval hippocampus with a horizontal long axis, a shape we classified as "oval." All other shapes were classified as "nonoval."

An oval hippocampus was observed as early as GW 21 in 1 fetus unilaterally, but all the other fetuses with an oval hippocampus were at least $27 \mathrm{GWs}$. A nonoval hippocampus was found at GW 24 at the earliest. Bilateral nonoval hippocampi were not observed after GW 29, and bilateral oval hippocampi were not observed before the same week. Our cohort is too small to definitely substantiate the hypothesis that the nonoval shape precedes the oval shape in the hippocampal development, but our data do not contradict that possibility. Furthermore, this pattern could also be seen in our previous sonographic study of preterm neonates, ${ }^{7}$ with a statistically significant difference between the frequencies of nonoval hippocampi before and after GW 25. The present study did not reveal such a threshold. This discrepancy may be due to the small numbers of the fetuses at that age and to the differences in methodology. The shape of the hippocampi was assessed in all cases in the sonographic study, but in the present study, that assessment was only made if the hippocampal sulcus was closed, and most the fetuses had open hippocampal sulci. We believe that hippocampi with nonoval shapes most likely represent IHI; indeed, some hippocampi never reach the complete inverted shape even in adulthood. ${ }^{12,13}$ The frequency of IHI has been shown to be as high as $14 \%-20 \%$ in preterm neonates at GWs 25-36 in the sonographic study ${ }^{7}$ and 19\% in MR imaging series of children and adults. ${ }^{12,13}$ These frequencies are best comparable with the frequency of the uni- or bilateral nonoval hippocampi in the fetuses in which both hippocampal sulci were closed (from GW 33 onward). That frequency was $33 \%$.

The development of the hippocampus, including the hippocampal sulcus and the inverted hippocampal formation, was often asymmetric in our series $(26 / 63,41 \%)$, and in a vast majority of these hippocampi, the right side $(23 / 26,88 \%)$ developed faster. In a prenatal MR imaging study, in which the hippocampal infolding angles were measured at GWs 20-37, this asymmetry was not observed. ${ }^{10}$ However, another group that used the same methodology in infants and children found a left-right asymmetry and concluded that it may result from slower development of the left hippocampus. ${ }^{19}$ Faster gyral development of the right cerebral hemisphere has been previously reported by Chi et $\mathrm{al},{ }^{20}$ who evaluated photographs of 507 formalin-fixed brains from 10 to 44 weeks' GA and sectioned 207 brains. They did not mention the hippocampal sulcus in the report. The recent MR imaging study of fetuses in utero by Habas et $\mathrm{al}^{21}$ also showed faster development of the right hemisphere, including the right parahippocampal gyrus. Electrophysiologic development is known to be slower in the left temporal lobe in healthy infants and children. ${ }^{22}$

The collateral sulcus was also evaluated in our study, even if it is not a part of the hippocampal formation itself. This sulcus also had a tendency to develop earlier on the right side, and it was identifiable as early as at GW 17, even if not visible in 1 fetus at GW 29. In the materials of Chi et $\mathrm{al}^{20}$ and Garel et al, ${ }^{9}$ the collateral sulcus was observed at the ages of 23 and $26 \mathrm{GW}$, respectively. The most recent MR imaging study by Habas et $\mathrm{al}^{21}$ has shown that the collateral sulcus is formed around GW 24. The orientation of the collateral sulcus can tend toward a vertical or a horizontal axis, the latter orientation being the most common in adults. A vertical orientation was seen unior bilaterally in $58 \%$ of the fetuses in our cohort that presented a well-defined sulcus, a slightly larger number $(P=.045)$ than the $36 \%$ described for children or adult populations. ${ }^{13}$ Also, this vertical orientation occurred more commonly on the left side, thus suggesting the possibility of a transitional phase toward a more mature horizontally oriented axis. However, this proposed hypothesis could not be confirmed within our own cohort because we failed to find any significant association between the GA and the percentage of vertical collateral sulci. Nevertheless, in children and adults, a nonoval hippocampal shape was always associated with a vertically oriented collateral sulcus. ${ }^{12}$ In $3 / 9$ fetuses with a nonoval hippocampus and a deep collateral sulcus, the collateral sulcus did not have a vertical orientation. This might be a sign that the hippocampus had not yet reached the oval shape but that the inversion process was continuing.

A weakness of the study is the low number of the fetuses, but the number is much higher than that in the earlier hippocampal studies on formalin-fixed brains. Repeated examinations of healthy fetuses would be the best method to evaluate normal developmental progress, but it is not ethically acceptable to conduct examinations on living fetuses without clinical indications.

\section{Conclusions}

There are large temporal variations in the development of the hippocampal sulcus, the hippocampal shape, and the collateral sulcus. The development is frequently asymmetric, with a faster development more often on the right side. Evaluation of normality in the fetal hippocampal region should thus be made with caution. The findings add to the understanding of the IHI found in the adult population and nearly always on the left side.

\section{References}

1. Kier EL, Fulbright RK, Bronen RA. Limbic lobe embryology and anatomy: dissection and MR of the medial surface of the fetal cerebral hemisphere. AJNR Am J Neuroradiol 1995; 16:1847-53

2. Kier EL, Kim JH, Fulbright RK, et al. Embryology of the human fetal hippocampus: MR imaging, anatomy, and histology. AJNR Am J Neuroradiol 1997; 18:525-32

3. Rados M, Judas M, Kostovic I. In vitro MRI of brain development. Eur J Radiol 2006;57:187-98

4. Humphrey T. The development of the human hippocampal fissure. J Anat 1967;101:655-76

5. Arnold SE, Trojanowski QJ. Human fetal hippocampal development, I. Cytoarchitecture, myeloarchitecture and neuronal morphologic features. J Comp Neurol 1996;367:274-92

6. Arnold SE, Trojanowski QJ. Human fetal hippocampal development. II. The neuronal cytoskeleton. J Comp Neurol 1996;367:293-307

7. Bajic D, Ewald U, Raininko R. Hippocampal development at gestation weeks 23 to 36: an ultrasound study on preterm neonates. Neuroradiology 2010;52: 489-94

8. Sasaki M, Sone M, Ehara S, et al. Hippocampal sulcus remnant: potential cause of change in signal intensity in the hippocampus. Radiology 1993;188:743-46

9. Garel C, Chantrel E, Elmaleh M, et al. Fetal MRI: normal gestational landmarks for cerebral biometry, gyration and myelination. Childs Nerv Syst 2003;19: $422-25$

10. Righini A, Zirpoli S, Parazzini C, et al. Hippocampal infolding angle changes during brain development assessed by prenatal MR imaging. AJNR Am J Neuroradiol 2006;27:2093-97 
11. Baker LL, Barkovich AJ. The large temporal horn: MR analysis in developmental brain anomalies versus hydrocephalus. AJNR Am J Neuroradiol 1992;13: $115-22$

12. Bajic D, Wang Ch, Kumlien E, et al. Incomplete inversion of the hippocampus: a common developmental anomaly. Eur Radiol 2008;18:138-42

13. Bajic D, Kumlien E, Mattsson P, et al. Hippocampal inversion: is there a relation to epilepsy? Eur Radiol 2009;19:2544-50

14. Almog B, Gamzu R, Achiron R, et al. Fetal lateral ventricular width: what should be its upper limit? A prospective cohort study and reanalysis of the current and previous data. J Ultrasound Med 2003;22:39-43

15. Farrell TA, Hertzberg BS, Kliewer MA, et al. Fetal lateral ventricles: reassessment of normal values for atrial diameter at US. Radiology 1994;193:409-11

16. Griffiths PD, Reeves MJ, Morris JE, et al. A prospective study of fetuses with isolated ventriculomegaly investigated by antenatal sonography and in utero MR imaging. AJNR Am J Neuroradiol 2010;31:106-11
17. Wyldes M, Watkinson M. Isolated mild fetal ventriculomegaly. Arch Dis Child Fetal Neonatal Ed 2004;89:F9-13

18. Snijders RJ, Nicolaides KH. Fetal biometry at $14-40$ weeks' gestation. Ultrasound Obstet Gynecol 1994;4:34-48

19. Okada Y, Kato T, Iwai K, et al. Evaluation of hippocampal infolding using magnetic resonance imaging. Neuroreport 2003;14:1405-09

20. Chi JG, Dooling EC, Gilles FH. Gyral development of the human brain. Ann Neurol 1977;1:86-93

21. Habas PA, Scott JA, Roosta A, et al. Early folding patterns and asymmetries of the normal human brain detected from in utero MRI. Cereb Cortex 2012;22:13-25

22. Petersen I, Eeg-Olofsson O. The development of the electroencephalogram in normal children from the age of 1 through 15 years: non-paroxysmal activity. Neuropediatrie 1971;2:247-304 